\title{
Nocturnal urinary melatonin excretion is associated with non-dipper pattern in elderly hypertensives
}

\author{
Kenji Obayashi ${ }^{1}$, Keigo Saeki ${ }^{1}$, Junko Iwamoto ${ }^{2}$, Nozomi Okamoto ${ }^{1}$, Kimiko Tomioka ${ }^{1}$, Satoko Nezu ${ }^{1}$, \\ Yoshito Ikada ${ }^{3}$ and Norio Kurumatani ${ }^{1}$
}

\begin{abstract}
Although oral melatonin administration may enhance a nocturnal blood pressure fall, it remains unclear whether endogenous melatonin, which is present at considerably lower levels than pharmacological melatonin, is associated with the non-dipper pattern. The present cross-sectional study aimed to determine the association between urinary melatonin excretion, an index of endogenous melatonin, and the non-dipper pattern. We measured the following variables in 141 elderly hypertensives: overnight urinary melatonin excretion, ambulatory blood pressure and actigraphic physical activity. We defined a non-dipper pattern as a $<10 \%$ fall in sleep systolic blood pressure compared with awake systolic blood pressure. When participants were divided into two groups (high and low melatonin groups) by the cutoff value for identifying the top tertile, the characteristics, except for age, did not significantly differ between the two groups. Crude logistic regression analysis showed significant associations of the non-dipper pattern with age, diabetes, higher urinary melatonin excretion (high vs. low) and daytime activity. In a multivariate analysis after adjustment for age, diabetes and daytime activity, the odds ratio for the non-dipper pattern in the high melatonin group was significantly lower than that in the low melatonin group (odds ratio: $0.39,95 \%$ confidence interval (Cl): $0.17-0.91$, $\boldsymbol{P}=0.03$ ). Moreover, the mean percentage systolic blood pressure nocturnal fall, adjusted for the former covariates, was significantly higher in the high melatonin group than the low melatonin group (difference $3.5 \%, 95 \% \mathrm{Cl}: 0.0-7.0 \%$, $P=0.048$ ). Among elderly hypertensive individuals, nocturnal urinary melatonin excretion is significantly and inversely
\end{abstract} associated with the non-dipper pattern.

Hypertension Research (2013) 36, 736-740; doi:10.1038/hr.2013.20; published online 11 April 2013

Keywords: circadian rhythm; elderly; hypertension; melatonin; non-dipper

\section{INTRODUCTION}

Hypertensive subjects who do not have a nocturnal blood pressure (BP) fall (non-dipper pattern) have more cardiovascular target organ damage $\mathrm{e}^{1-3}$ and worse cardiovascular outcomes ${ }^{4-6}$ than those who do (dipper pattern). The non-dipper pattern is observed in approximately half of the hypertensive population and is caused by old age, high body mass index (BMI) and diabetes, ${ }^{7}$ and the clinical importance of the non-dipper pattern increases in hypertensive individuals. $^{8}$

Melatonin ( $\mathrm{N}$-acetyl-5-methoxytryptamine) is a pineal gland hormone secreted predominantly at night. Nocturnal serum melatonin production is accurately reflected by nocturnal urinary excretion of the major metabolite of melatonin, 6-sulfatoxymelatonin, after rapid metabolism. ${ }^{9}$ In addition, melatonin plays an important role in BP reduction, including enhancement of vasodilation, reduction in myocardial contractility and decreased sympathetic activity. ${ }^{10-12}$ Several clinical trials have suggested that oral melatonin administration enhances nocturnal BP fall in hypertensive subjects, ${ }^{13,14}$ but it remains unclear whether endogenous melatonin, which is present at considerably lower levels than pharmacological melatonin, ${ }^{15}$ is associated with the non-dipper pattern.

The purpose of this observational study was to clarify the association between urinary 6-sulfatoxymelatonin excretion (UME), an index of endogenous melatonin, and the non-dipper pattern in elderly hypertensives.

\section{METHODS}

Participants

We studied 141 elderly hypertensive individuals who were consecutively selected from among 211 subjects, voluntarily recruited and who met the following inclusion criteria: those who were aged $\geqslant 60$ years; those who had hypertension diagnosed with an ambulatory awake BP of $\geqslant 140 / 90 \mathrm{~mm} \mathrm{Hg}$ an ambulatory sleep BP of $\geqslant 125 / 75 \mathrm{~mm} \mathrm{Hg}$; and/or if participants had a previous diagnosis of hypertension and were receiving current antihypertensive medication. ${ }^{16}$ Of these individuals, 70 were excluded from the analysis because of the following reasons: those who were normotensive on ambulatory BP monitoring $(\mathrm{ABPM})$ without receiving current antihypertensive medication

${ }^{1}$ Department of Community Health and Epidemiology, Nara Medical University School of Medicine, Nara, Japan; ${ }^{2}$ Department of Nursing, Tenri Health Care University, Nara, Japan and ${ }^{3}$ Department of Surgery, Nara Medical University School of Medicine, Nara, Japan

Correspondence: Dr K Obayashi, Department of Community Health and Epidemiology, Nara Medical University School of Medicine, 840 Shijocho, Kashiharashi, Nara 6348521 , Japan.

E-mail: obayashi@naramed-u.ac.jp

Received 10 August 2012; revised 9 January 2013; accepted 9 January 2013; published online 11 April 2013 
( $n=60$ ), those whose sleep period was not detected by actigraphy $(n=5)$, those whose BP data were lost for $>25 \%$ of the study period because of errors or artifacts $(n=3)$, those whose urine was not collected according to our protocol $(n=1)$ and those who consumed alcohol during the designated monitoring period $(n=1)$. Written informed consent was obtained from all participants in the study.

\section{Study protocol}

We visited each participant's home between September 2010 and April 2011 and collected demographic and medical information including age, gender, medical history and medication during a personal interview. A standardized questionnaire was administered by a trained interviewer. A venous sample was collected during the visit, and ABPM and actigraphic monitoring were performed from the noon of day 1 to the noon of day 3 (Figure 1). Participants were instructed to collect their urine during the night of the second day. Finally, we revisited each home to retrieve our instruments and collect the urine samples. All participants were advised against alcohol consumption during the designated monitoring period. The study protocol was approved by the ethical committee of Nara Medical University.

\section{Urinary 6-sulfatoxymelatonin excretion}

Participants were instructed to follow a collection protocol that required discarding the last void at bedtime and collecting urine from the time of going to sleep to the first morning void. The overnight urine sample was kept at room temperature, avoiding direct sunlight, until collection. The total volume of urine collected overnight was measured upon sample collection and then stored at $-20^{\circ} \mathrm{C}$ until assay. The urinary concentration of 6-sulfatoxymelatonin was measured by a commercial laboratory (SRL, Tokyo, Japan) using a highly sensitive enzyme-linked immunosorbent assay kit (RE54031; IBL, Hamburg, Germany) with a lower 6-sulfatoxymelatonin detection limit of $1.0 \mathrm{ng} \mathrm{ml}^{-1}$. None of the participants had 6-sulfatoxymelatonin levels below the detection limit for assay. UME was calculated as follows:

UME $(\mu \mathrm{g})=$ concentration of 6 -sulfatoxymelatonin $\left(\mu \mathrm{g} \mathrm{ml}^{-1}\right) \times$ total volume of overnight urine $(\mathrm{ml})$

The reproducibility of UME among 36 participants was assessed by additional collection of urine samples 4 months later; the intraclass correlation coefficient (ICC) was evaluated for agreement after natural log transformation of UME values because of a skewed distribution. The ICC between two UME levels was 0.77 (95\% confidence interval (CI): 0.60-0.88).

\section{Ambulatory BP monitoring}

ABPM was performed on the non-dominant arm using a validated ambulatory recorder (TM-2430; A\&D, Tokyo, Japan) and suitably sized cuff. We measured BP from the noon of day 1 to the noon of day 3 at 30-min intervals for $48 \mathrm{~h}$. Recordings taken during the awake and sleep periods of the second day were used for analysis. Erroneous BP data were excluded as were values with systolic $\mathrm{BP}$ (SBP) $<50$ or $>250 \mathrm{~mm} \mathrm{Hg}$ and diastolic BP $<30$ or $>160 \mathrm{~mm} \mathrm{Hg}$. We estimated the awake and sleep periods by actigraphy. We defined a non-dipper pattern as a $<10 \%$ fall in sleep SBP compared with awake SBP. ${ }^{17}$ Among the 201 subjects (141 hypertensives and 60 normotensives), 44\% used an antihypertensive drug, the mean 24-h ambulatory BP was $129 / 76 \mathrm{~mm} \mathrm{Hg}$ and $33 \%$ had a non-dipper pattern.

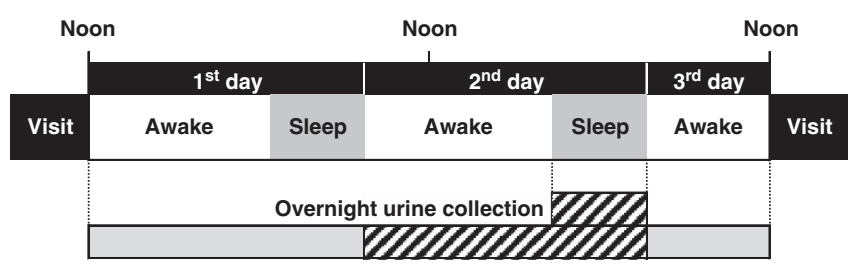

Ambulatory BP monitoring \& Actigraphic monitoring

Figure 1 Data collection schedule: ambulatory blood pressure (BP) monitoring and actigraphic monitoring were performed from the noon of day 1 to the noon of day 3. Overnight urine was collected during the sleep period of the second day. Data from the shaded areas were used for analysis.

\section{Actigraphic monitoring}

Actigraphic parameters were measured using an actigraph (Actiwatch 2; Respironics, Murrysville, PA, USA) to detect movement; we preferred to perform actigraphic monitoring on the non-dominant wrist because the dominant arm shows overcounts for physical activity by minor activity with trunk still (for example, writing, cooking and brushing teeth). ${ }^{18}$ The internal clocks of the ABPM device and actigraph were synchronized. We began and completed measurements on both devices simultaneously; actigraphic measurements were performed at 1-min intervals for $48 \mathrm{~h}$. Recordings taken during the awake and sleep periods of the second day were used for analysis. Three actigraphic parameters were determined by Actiware version 5.5 (Respironics) with a default wake threshold value of 40 (moderate) and 10 immobile min for sleep onset to identify individual epochs as awake or sleep: ${ }^{19}$ sleep duration (total duration of sleep), sleep efficiency (calculated by dividing total sleep time by total rest time) and physical activity (average of all valid physical activity counts per min).

\section{Other measurements}

Body weight and height were measured, and BMI was calculated as weight $(\mathrm{kg}) /$ height $\left(\mathrm{m}^{2}\right)$. Current smoking and habitual drinking status were evaluated by self-report questionnaire. Venous samples were analyzed by a commercial laboratory (SRL) using a standard clinical chemistry analyzer to determine hemoglobin Alc levels and concentrations of fasting plasma glucose and creatinine. Diabetes mellitus was diagnosed using medical history, current antidiabetic treatment or if fasting plasma glucose was $\geqslant 7.0 \mathrm{mmoll}^{-1}$ and the hemoglobin Alc level was $\geqslant 6.5 \%$ of the National Glycohemoglobin Standardization Program value. The estimated glomerular filtration rate (eGFR) was calculated by the Japanese Society of Nephrology-Chronic Kidney Disease Practice Guide formula: eGFR $\left(\mathrm{ml} \mathrm{min}^{-1}\right.$ per $\left.1.73 \mathrm{~m}^{2}\right)=194 \times($ serum creatinine $\left.\left(\mathrm{mg} \mathrm{dl}^{-1}\right)\right)^{-1.094} \times(\text { age }(\text { years }))^{-0.287}$. The result was multiplied by a correction factor of 0.739 for women.

\section{Statistical analysis}

Mean \pm s.d. values were reported for variables with a normal distribution, and median and interquartile ranges for variables with an asymmetrical distribution. The study participants were divided into two groups (high and low melatonin groups) by the cutoff value for identifying the top tertile, and comparisons between high and low UME groups were performed using unpaired $t$-tests for continuous data with a normal distribution, the MannWhitney test for continuous data with an asymmetrical distribution and the $\chi^{2}$ test for categorical data. Comparisons between non-dipper and dipper pattern were also performed. Crude odds ratios (ORs) with 95\% CI of the non-dipper pattern were estimated using a logistic regression model. In the model, explanatory variables included age (per year), gender (male or female), BMI $\left(\mathrm{kg} \mathrm{m}^{-2}\right.$ ), obesity (BMI $\geqslant 25$ or $<25 \mathrm{~kg} \mathrm{~m}^{-2}$ ), current smoker (yes or no), habitual drinker (consume alcohol daily or not), use of antihypertensive medication (yes or no), evening administration of antihypertensive drug (yes or no), diabetes (yes or no), UME (high or low), eGFR ( $\mathrm{ml} \mathrm{min}^{-1}$ per $1.73 \mathrm{~m}^{2}$ ), sleep duration (per min), sleep efficiency (\%), poor quality of sleep, that is, sleep efficiency $<70 \%$ or total sleep time $<5 \mathrm{~h}$ (yes or no), daytime activity (counts per min) and nocturnal activity (counts per min). ORs were adjusted simultaneously for variables that showed a significant association with the non-dipper pattern in the univariate model. In addition, the percentage SBP nocturnal fall was adjusted for variables significantly associated with the non-dipper pattern in the univariate model using analysis of covariance. We evaluated trends in continuous data using the Jonckheere-Terpstra test for trends. ${ }^{20}$ Statistical analyses were performed using SPSS for Windows version 19.0 (IBM SPSS Inc., Chicago, IL, USA), and statistical significance was set at the two-sided $P$-value of $<0.05$.

\section{RESULTS}

Of the 141 participants with hypertension, the median UME was $7.7 \mu \mathrm{g}$ (interquartile range 4.6-11.0). The cutoff value for identifying the top tertile (the high UME group) was $9.7 \mu \mathrm{g}$. The characteristics of the 141 hypertensives, divided into two groups (the high and low 
Table 1 Characteristics of 141 hypertensives stratified by urinary 6-sulfatoxymelatonin excretion (UME) levels

\begin{tabular}{|c|c|c|c|}
\hline \multirow[b]{2}{*}{ Characteristics } & High UME group & Low UME group & \multirow[b]{2}{*}{ P-valu } \\
\hline & $(n=47)$ & $(n=94)$ & \\
\hline Age, mean, years & $69.2(4.9)$ & $71.4(7.1)$ & 0.04 \\
\hline Gender, number, male & $25(53.2)$ & $45(47.9)$ & 0.55 \\
\hline Body mass index, mean, $\mathrm{kg} \mathrm{m}^{-2}$ & $23.5(3.0)$ & $22.6(3.1)$ & 0.10 \\
\hline Obesity $\left(\geqslant 25 \mathrm{~kg} \mathrm{~m}^{-2}\right)$, number & $13(27.7)$ & $23(24.5)$ & 0.14 \\
\hline Current smoker, number & $1(2.1)$ & $8(8.5)$ & 0.14 \\
\hline Habitual drinker, number & $12(25.6)$ & $23(24.5)$ & 0.89 \\
\hline UME, median, $\mu \mathrm{g}$ & $13.5(11.0-16.8)$ & $6.0(3.4-7.8)$ & $<0.00$ \\
\hline \multicolumn{4}{|l|}{ Actigraphic parameters } \\
\hline Sleep duration, mean, min & $460.9(87.3)$ & $451.2(104.0)$ & 0.55 \\
\hline Sleep efficiency, mean, \% & $85.8(6.4)$ & $83.6(8.2)$ & 0.11 \\
\hline Poor sleep quality, number & $5(10.6)$ & $15(16.0)$ & 0.39 \\
\hline
\end{tabular}

Data are means (s.d.), medians (interquartile range) or number (\%). Habitual drinker is one who drinks any alcohol every day. Poor sleep quality is poor sleep efficiency $<70 \%$ or total sleep time $<5$.

UME groups), are presented in Table 1. Participants in the high UME group were younger than those in the low UME group. Table 2 shows the characteristics stratified by circadian BP status (non-dipper and dipper pattern). Hypertensives with the dipper pattern were significantly younger, and had a lower frequency of diabetes, a higher UME and higher daytime activity than those with the non-dipper pattern. In addition, hypertensives with the dipper pattern showed a significantly lower sleep BP than those with the non-dipper pattern. The ICCs of percentage SBP nocturnal fall and circadian BP status were 0.46 and 0.38 , respectively. None of the participants had an eGFR value $<30 \mathrm{ml} \mathrm{min}^{-1}$ per $1.73 \mathrm{~m}^{2}$, and none consumed any drug or supplement containing melatonin.

Crude logistic regression analysis showed significant associations between non-dipper pattern and age, diabetes, higher UME and daytime activity. Multivariate analysis adjusted for age, diabetes and daytime activity indicated that the OR for the non-dipper pattern was significantly lower in the high UME group than in the low UME group (OR: 0.39, 95\% CI: 0.17-0.91, $P=0.03$; Table 3).

The mean percentage SBP nocturnal fall was marginally higher in the high UME group than in the low UME group $(14.4 \pm 9.8 \%$ and $11.4 \pm 10.5 \%$, respectively; $P=0.10$ ). In multivariate analysis, mean percentage SBP nocturnal fall adjusted for age, diabetes and daytime activity was significantly higher in the high UME group than in the low UME group (difference: 3.5, 95\% CI: $0.0-7.0 \%, P=0.048$; Table 4).

In addition, a marked and consistent difference in UME was observed in categories based on nocturnal SBP fall: extreme dipper pattern $(n=28)$, nocturnal SBP fall $\geqslant 20 \%$; normal dipper pattern $(n=61)$, nocturnal SBP fall $<20 \%$ but $\geqslant 10 \%$; decreased dipper pattern $(n=36)$, nocturnal SBP fall $<10 \%$ but $\geqslant 0 \%$; and reverse dipper pattern $(n=16)$, nocturnal SBP fall $<0 \%$. UME was highest in hypertensive individuals with the extreme dipper pattern and lowest in those with the reverse dipper pattern $\left(P_{\text {trend }}=0.02\right.$; Figure 2); 11 hypertensives (39\%) from the extreme dipper pattern population were included in the high UME group.

\section{DISCUSSION}

This study demonstrated that UME is significantly and inversely associated with the non-dipper pattern in elderly hypertensives.
Table 2 Clinical, actigraphic and BP characteristics of 141 hypertensives by circadian BP status

\begin{tabular}{|c|c|c|c|}
\hline \multirow[b]{2}{*}{ Characteristics } & Non-dipper & Dipper & \multirow[b]{2}{*}{ P-value } \\
\hline & $(\mathrm{n}=52)$ & $(\mathrm{n}=89)$ & \\
\hline Age, mean, years & $72.5(6.6)$ & $69.6(6.3)$ & 0.01 \\
\hline Gender, number, male & $24(46.2)$ & $46(51.7)$ & 0.53 \\
\hline Body mass index, mean, $\mathrm{kg} \mathrm{m}^{-2}$ & $22.3(3.0)$ & $23.2(3.1)$ & 0.10 \\
\hline Obesity $\left(\geqslant 25 \mathrm{~kg} \mathrm{~m}^{-2}\right)$, number & $9(17.3)$ & $20(22.5)$ & 0.46 \\
\hline Current smoker, number & $5(9.6)$ & $4(4.5)$ & 0.23 \\
\hline Habitual drinker, number & $12(23.1)$ & $23(25.8)$ & 0.71 \\
\hline $\begin{array}{l}\text { Use of antihypertensive medication, } \\
\text { number }\end{array}$ & $28(53.8)$ & $60(67.4)$ & 0.11 \\
\hline $\begin{array}{l}\text { Evening use of antihypertensive drug, } \\
\text { number }\end{array}$ & $7(13.5)$ & $18(20.2)$ & 0.31 \\
\hline Diabetes, number & $15(28.8)$ & $12(13.5)$ & 0.03 \\
\hline UME, median, $\mu \mathrm{g}$ & $7.1(3.5-9.3)$ & $8.7(5.0-12.0)$ & 0.04 \\
\hline eGFR, mean, $\mathrm{ml} \mathrm{min}^{-1}$ per $1.73 \mathrm{~m}^{2}$ & $68.9(13.3)$ & $73.2(14.8)$ & 0.08 \\
\hline \multicolumn{4}{|l|}{ Actigraphic parameters } \\
\hline Sleep duration, mean, min & $453.9(105.4)$ & $454.8(94.9)$ & 0.96 \\
\hline Sleep efficiency, mean, \% & $83.4(8.3)$ & $84.8(7.3)$ & 0.32 \\
\hline Poor sleep quality, number & $8(15.4)$ & $12(13.5)$ & 0.76 \\
\hline $\begin{array}{l}\text { Daytime activity, mean, } \\
\text { counts per min }\end{array}$ & $275.7(98.7)$ & 312.6 (106.9) & 0.04 \\
\hline $\begin{array}{l}\text { Nocturnal activity, median, } \\
\text { counts per min }\end{array}$ & $17.8(8.6)$ & $16.5(12.5)$ & 0.52 \\
\hline \multicolumn{4}{|c|}{ Ambulatory BP parameters (mean, $\mathrm{mm} \mathrm{Hg}$ ) } \\
\hline Awake SBP & $137.3(16.4)$ & $141.0(14.6)$ & 0.17 \\
\hline Awake DBP & $80.0(9.1)$ & $82.8(7.5)$ & 0.04 \\
\hline Sleep SBP & $134.4(15.5)$ & $114.7(13.7)$ & $<0.001$ \\
\hline Sleep DBP & $74.8(8.2)$ & $67.6(7.9)$ & $<0.001$ \\
\hline 24-h SBP & $134.3(15.8)$ & $132.7(13.9)$ & 0.54 \\
\hline 24-h DBP & $78.1(8.5)$ & $77.9(6.8)$ & 0.85 \\
\hline
\end{tabular}

Abbreviations: BP, blood pressure; DBP, diastolic blood pressure; eGFR, estimated glomerular filtration rate; SBP, systolic blood pressure; UME, urinary 6-sulfatoxymelatonin excretion. Data are means (s.d.), medians (interquartile range) or number (\%). Habitual drinker is one who drinks any alcohol every day. Poor sleep quality is poor sleep efficiency $<70 \%$ or total sleep time $<5 \mathrm{~h}$.

Evidence included the fact that the OR for the non-dipper pattern in the high UME group (the top tertile) is significantly lower than that in the low UME group after adjustment for age, diabetes and daytime activity (OR: 0.39 , 95\% CI: $0.17-0.91, P=0.03$ ). In addition, this finding is supported by the consistent association observed between UME and percentage SBP nocturnal fall (difference: 3.5\%, 95\% CI: $0.0-7.0 \%, P=0.048$ ).

Our results suggest an inverse relationship between endogenous melatonin and the non-dipper pattern in a smaller difference in melatonin levels relative to oral melatonin administration. Although several clinical trials have suggested that oral melatonin administration enhances nocturnal BP fall in hypertensive subjects, ${ }^{13,14}$ pharmacological melatonin levels even at a low dosage $(2 \mathrm{mg})$ and with prolonged-release tablets vary widely and are 10-fold higher than endogenous melatonin levels. ${ }^{15,21}$ In our study, the median UME of the high UME group was 2.3-fold as high as the low UME group, and thus we indicate the effect of endogenous melatonin on the non-dipper pattern in a smaller difference in melatonin levels relative to oral melatonin administration.

To our knowledge, only two previous observational studies have reported that lower melatonin production influences the non-dipper 
Table 3 Crude and adjusted odds ratios for non-dipper pattern

\begin{tabular}{|c|c|c|c|c|}
\hline Covariates & Crude OR $(95 \% \mathrm{Cl})$ & P-value & Adjusted ORa $(95 \% \mathrm{Cl})$ & P-value \\
\hline Age (per year) & $1.07(1.02-1.13)$ & 0.01 & $1.06(1.00-1.12)$ & 0.054 \\
\hline Gender (male vs. female) & $0.80(0.40-1.59)$ & 0.53 & - & - \\
\hline Obesity ( $\geqslant 25$ vs. $<25 \mathrm{~kg} \mathrm{~m}^{-2}$ ) & $0.72(0.30-1.73)$ & 0.47 & - & - \\
\hline Current smoker (yes vs. no ) & $2.26(0.58-8.83)$ & 0.24 & - & - \\
\hline Habitual drinker (yes vs. no) & $0.86(0.39-1.92)$ & 0.71 & - & - \\
\hline Diabetes (yes vs. no) & $2.60(1.11-6.11)$ & 0.03 & $2.92(1.17-7.28)$ & 0.02 \\
\hline Higher UME (high vs. low) & $0.40(0.18-0.87)$ & 0.02 & $0.39(0.17-0.91)$ & 0.03 \\
\hline eGFR (per ml min ${ }^{-1}$ per $1.73 \mathrm{~m}^{2}$ ) & $0.98(0.96-1.00)$ & 0.08 & - & - \\
\hline \multicolumn{5}{|l|}{ Actigraphic parameters } \\
\hline Sleep duration (per min) & $1.00(1.00-1.00)$ & 0.96 & - & - \\
\hline
\end{tabular}

Abbreviations: $\mathrm{CI}$, confidence interval; eGFR, estimated glomerular filtration rate; OR, odds ratio; UME, urinary 6-sulfatoxymelatonin excretion.

Habitual drinker is one who drinks any alcohol every day. Poor sleep quality is poor sleep efficiency $<70 \%$ or total sleep time $<5 \mathrm{~h}$.

${ }^{a}$ Adjusted for all covariates shown.

Table 4 Adjusted percentage SBP nocturnal fall between the high and low UME groups

\begin{tabular}{cccc}
$\begin{array}{c}\text { High UME group } \\
(\mathrm{n}=47)\end{array}$ & $\begin{array}{c}\text { Low UME group } \\
(\mathrm{n}=94)\end{array}$ & \\
\cline { 2 - 3 } & Mean & Difference & \\
Mean & $(95 \% \mathrm{Cl})$ & $(95 \% \mathrm{Cl})$ & P-value \\
\hline
\end{tabular}

$\begin{array}{lllll}\text { Percentage SBP } & 14.7(11.9-17.5) & 11.2(9.2-13.2) & 3.5(0.0-7.0) & 0.048\end{array}$

nocturnal fall $(\%)^{a}$

Abbreviations: $\mathrm{Cl}$, confidence interval; SBP, systolic blood pressure; UME, urinary 6-sulfatoxymelatonin excretion.

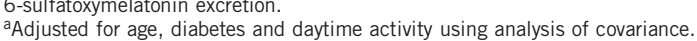

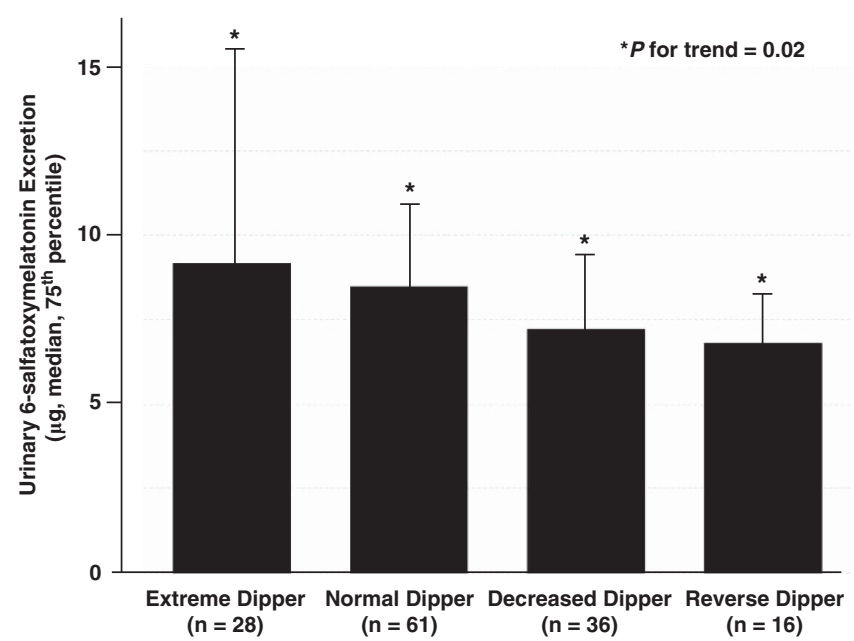

Figure 2 Consistent difference in urinary melatonin excretion based on category of nocturnal systolic blood pressure (SBP) fall. The $P$-value is shown for the trend identified by the Jonckheere-Terpstra test. ${ }^{20}$ Solid bars indicate the median urinary melatonin excretion, and error bars indicate the upper half of the interquartile range (75th percentile). pattern. One study showed that the night-to-day ratio of plasma melatonin levels was lower in hypertensive patients with the non-dipper pattern compared with those with the dipper pattern. ${ }^{22}$ The other study reported that nocturnal increase in urinary melatonin levels was impaired in hypertensive patients with the non-dipper pattern. ${ }^{23}$ However, the confounding effects of other causes of the non-dipper pattern, such as age, BMI and diabetes, ${ }^{7}$ were not adequately considered in these studies; hence, it was unclear whether endogenous melatonin was associated with the non-dipper pattern independent of potential confounders. Our results indicate that among the elderly hypertensive population, UME is inversely associated with the non-dipper pattern, independent of age, diabetes and daytime activity.

The potential mechanisms of melatonin's effect on BP have been studied under varying experimental conditions. Melatonin increases cytosolic $\mathrm{Ca}^{2+}$ in endothelial cells, increases levels of nitric oxide and decreases levels of reactive oxygen species. ${ }^{24,25}$ Melatonin also influences the enhancement of vasodilation and reduction of myocardial contractility, and lowers BP and serum norepinephrine levels. ${ }^{10,11,26}$ Pinealectomy enhances peripheral vasoconstriction and results in hypertension, which can be reversed by melatonin administration in rats. ${ }^{27}$ In humans, BP and norepinephrine levels decrease following melatonin administration. ${ }^{12}$

The highest levels of UME were observed in elderly hypertensives who displayed the extreme dipper pattern, which BP pattern was associated with a worse prognosis in a previous study. Melatonin is a scavenger of free radicals, and may protect against atherosclerosis and oxidative stress. ${ }^{21,28}$ In fact, a recent report suggested that higher urinary melatonin levels are associated with a lower incidence of hypertension among young women. ${ }^{29}$ Although a previous study reported that elderly hypertensive patients with the extreme dipper pattern had a worse prognosis than those with the dipper pattern, ${ }^{6}$ $39 \%$ of elderly hypertensives who displayed the extreme dipper pattern had the highest UME levels in our study. The discrepancy from the prognostic point of view is that a considerable number of elderly hypertensives with the extreme dipper pattern had the highest 
levels of UME in our study. Further prospective study of melatonin's effect on prognosis is necessary to clarify this discrepancy.

The present study has several limitations. First, the target population was not randomly selected, and hence there is a possibility of selection bias (participation rate unknown). Our 201 subjects (141 hypertensives and 60 normotensives) had mildly higher 24-h ambulatory $\mathrm{BP}$ and took more antihypertensive medications than participants in the Ohasama Japanese population-based study (129/76 vs. $123 / 72 \mathrm{~mm} \mathrm{Hg}$ and $44 \%$ vs. $31 \%$, respectively). ${ }^{8}$ However, as the prevalence of the non-dipper pattern was similar between the two studies (33\% vs. $35 \%$, respectively), the generalizability of the present findings may be acceptable given that the participants in our study were 10 years older than those in the Ohasama study. In addition, the 141 hypertensives were included according to different criteria: based on a diagnosis derived from ABPM or from a previous diagnosis of hypertension and current antihypertensive medication based on a previous clinic BP, although these previous clinic BP data were not available. Second, we assessed a single overnight urine sample rather than multiple samples; therefore, the melatonin status of some participants was probably misclassified. However, the ICC between two UME levels in our study was 0.77, suggesting that serious misclassification was rare. Moreover, we measured only nocturnal melatonin levels in this study; therefore, we could not evaluate the association between circadian BP pattern and daytime or night-to-day change of melatonin levels. Further research including whole day urine sample is needed. Finally, we evaluated sleep quality by actigraphy and may ignore the residual confounding effect of obstructive sleep apnea (OSA). OSA is an important condition that could affect the incidence of the non-dipper pattern, and high BMI is the most important risk factor for OSA. ${ }^{30}$ In our study, the mean BMI, prevalence of obesity (BMI $\geqslant 25 \mathrm{~kg} \mathrm{~m}^{-2}$ ) and prevalence of poor sleep quality (sleep efficiency $<70 \%$ or total sleep time $<5 \mathrm{~h}$ ), which is a predictor of sleep-disordered breathing including OSA, ${ }^{31}$ did not significantly differ between the high and low UME groups. In addition, we did not perform the screening test for secondary hypertension or orthostatic hypotension in this study.

In conclusion, among elderly hypertensive individuals, UME is inversely associated with the non-dipper pattern independently of age, diabetes and daytime activity. This finding is supported by the consistent association observed between UME and percentage SBP nocturnal fall.

\section{CONFLICT OF INTEREST}

The authors declare no conflict of interest.

\section{ACKNOWLEDGEMENTS}

We are indebted to all participants of this study. We thank our staff members for their valuable support during the survey. This study was supported by Grants from the Department of Indoor Environmental Medicine, Nara Medical University; Scientific Research (22790567) from the Ministry of Education, Culture, Sports, Science and Technology; Mitsui Sumitomo Insurance Welfare Foundation; and Meiji Yasuda Life Foundation of Health and Welfare.

1 Cuspidi C, Giudici V, Negri F, Sala C, Mancia G. Left ventricular geometry, ambulatory blood pressure and extra-cardiac organ damage in untreated essential hypertension. Blood Press Monit 2010; 15: 124-131.

2 Nagai M, Hoshide S, Ishikawa J, Shimada K, Kario K. Ambulatory blood pressure as an independent determinant of brain atrophy and cognitive function in elderly hypertension. J Hypertens 2008; 26: 1636-1641.
3 Routledge FS, McFetridge-Durdle JA, Dean CRCanadian Hypertension Society. Nighttime blood pressure patterns and target organ damage: a review. Can J Cardiol 2007, 23: $132-138$

4 Fagard RH, Celis H, Thijs L, Staessen JA, Clement DL, De Buyzere ML, De Bacquer DA Daytime and nighttime blood pressure as predictors of death and cause-specific cardiovascular events in hypertension. Hypertension 2008; 51: 55-61.

5 Boggia J, Li Y, Thijs L, Hansen TW, Kikuya M, Björklund-Bodegård K, Richart T, Ohkubo T, Kuznetsova T, Torp-Pedersen C, Lind L, Ibsen H, Imai Y, Wang J, Sandoya E, O'Brien E, Staessen JA. Prognostic accuracy of day versus night ambulatory blood pressure: a cohort study. Lancet 2007; 370: 1219-1229.

6 Kario K, Pickering TG, Matsuo T, Hoshide S, Schwartz JE, Shimada K. Stroke prognosis and abnormal nocturnal blood pressure falls in older hypertensives. Hypertension 2001; 38: 852-857.

7 de la Sierra A, Redon J, Banegas JR, Segura J, Parati G, Gorostidi M, de la Cruz JJ, Sobrino J, Llisterri JL, Alonso J, Vinyoles E, Pallarés V, Sarría A, Aranda P, Ruilope LM. Prevalence and factors associated with circadian blood pressure patterns in hypertensive patients. Hypertension 2009; 53: 466-472.

8 Ohkubo T, Hozawa A, Yamaguchi J, Kikuya M, Ohmori K, Michimata M, Matsubara M, Hashimoto J, Hoshi H, Araki T, Tsuji I, Satoh H, Hisamichi S, Imai Y. Prognostic significance of the nocturnal decline in blood pressure in individuals with and without high 24-h blood pressure: the Ohasama study. J Hypertens 2002; 20: 2183-2189.

9 Baskett JJ, Cockrem JF, Antunovich TA. Sulphatoxymelatonin excretion in older people relationship to plasma melatonin and renal function. J Pineal Res 1998; 24: 58-61.

10 Girouard H, Chulak C, Lejossec M, Lamontagne D, de Champlain J. Vasorelaxant effects of the chronic treatment with melatonin on mesenteric artery and aorta of spontaneously hypertensive rats. J Hypertens 2001; 19: 1369-1377.

11 Abete P, Bianco S, Calabrese C, Napoli C, Cacciatore F, Ferrara N, Rengo F. Effects of melatonin in isolated rat papillary muscle. FEBS Lett 1997; 412: 79-85.

12 Arangino S, Cagnacci A, Angiolucci M, Vacca AM, Longu G, Volpe A, Melis GB. Effects of melatonin on vascular reactivity, catecholamine levels, and blood pressure in healthy men. Am J Cardiol 1999; 83: 1417-1419.

13 Scheer FA, Van Montfrans GA, van Someren EJ, Mairuhu G, Buijs RM. Daily nighttime melatonin reduces blood pressure in male patients with essential hypertension. Hypertension 2004; 43: 192-197.

14 Grossman E, Laudon M, Yalcin R, Zengil H, Peleg E, Sharabi Y, Kamari Y, Shen-Orr Z, Zisapel N. Melatonin reduces night blood pressure in patients with nocturna hypertension. Am J Med 2006; 119: 898-902.

15 European Medicines Agency, Circadin EPA Report, 2007; available at http://www. ema.europa.eu/docs/en_GB/document_library/EPAR_-_Scientific_Discussion/human/ 000695/WC500026808.pdf (accessed on 6 February 2012)

16 Pickering TG, Hall JE, Appel LJ, Falkner BE, Graves J, Hill MN, Jones DW, Kurtz T, Sheps SG, Roccella EJ. Recommendations for blood pressure measurement in humans and experimental animals: Part 1: blood pressure measurement in humans: a statement for professionals from the Subcommittee of Professional and Public Education of the American Heart Association Council on High Blood Pressure Research.. Hypertension 2005; 45: 142-161.

17 Takakuwa H, Ise T, Kato T, Izumiya Y, Shimizu K, Yokoyama H, Kobayashi KI. Diurnal variation of hemodynamic indices in non-dipper hypertensive patients. Hypertens Res 2001; 24: 195-201.

18 Middelkoop HA, van Dam EM, Smilde-van den Doel DA, Van Dijk G. 45-hour continuous quintuple-site actimetry: relations between trunk and limb movements and effects of circadian sleep-wake rhythmicity. Psychophysiology 1997; 34: 199-203.

19 Philips Respironics Actiware Tutorials; available at http://learnactiware.com/tutorials/ (accessed on 20 March 2013)

20 Bewick V, Cheek L, Ball J. Statistics review 10: Further nonparametric methods Crit Care. 2004; 8: 196-199.

21 Brzezinski A. Melatonin in humans. N Engl J Med. 1997; 336: 186-195.

22 Zeman M, Dulková K, Bada V, Herichová I. Plasma melatonin concentrations in hypertensive patients with the dipping and non-dipping blood pressure profile. Life Sci 2005; 76: 1795-1803.

23 Jonas M, Garfinkel D, Zisapel N, Laudon M, Grossman E. Impaired nocturnal melatonin secretion in non-dipper hypertensive patients. Blood Press 2003; 12: 19-24.

24 Pogan L, Bissonnette P, Parent L, Sauvé R. The effects of melatonin on $\mathrm{Ca}^{2}$ homeostasis in endothelial cells. J Pineal Res 2002; 33: 37-47.

25 Anwar MM, Meki AR, Rahma HH. Inhibitory effects of melatonin on vascular reactivity: possible role of vasoactive mediators. Comp Biochem Physiol C Toxicol Pharmacol 2001; 130: 357-367.

26 K-Laflamme A, Wu L, Foucart S, de Champlain J. Impaired basal sympathetic tone and $\alpha 1$-adrenergic responsiveness in association with the hypotensive effect of melatonin in spontaneously hypertensive rats. Am J Hypertens 1998; 11: 219-229.

27 Holmes SW, Sugden D. The effect of melatonin on pinealectomy-induced hypertension in the rat. Br J Pharmacol 1976; 56: 360-361.

28 Reiter RJ, Melchiorri D, Sewerynek E, Poeggeler B, Barlow-Walden L, Chuang J, Ortiz GG, Acuña-Castroviejo D. A review of the evidence supporting melatonin's role as an antioxidant. J Pineal Res 1995; 18: 1-11.

29 Forman JP, Curhan GC, Schernhammer ES. Urinary melatonin and risk of incident hypertension among young women. J Hypertens 2010; 28: 446-451.

30 Pankow W, Nabe B, Lies A, Kohl FV, Lohmann FW. Influence of obstructive sleep apnoea on circadian blood pressure profile. J Sleep Res 1995; 4: 102-106.

31 Mehra R, Stone KL, Ancoli-Israel S, Litwack-Harrison S, Ensrud KE, Redline S. Interpreting wrist actigraphic indices of sleep in epidemiologic studies of the elderly: the Study of Osteoporotic Fractures. Sleep 2008; 31: 1569-1576. 